\title{
EL CONCEPTO DE ESQUEMA CORPORAL EN NIÑAS Y NIÑOS CON Y SIN NECESIDADES EDUCATIVAS ESPECIALES, DE PREESCOLAR, SEGUNDO Y CUARTO GRADOS
}

\author{
Zulay Pereira Pérez ${ }^{1}$ \\ Angélica Fontana Hernández ${ }^{2}$ \\ Dinia Rojas Rodríguez ${ }^{3}$
}

\section{Resumen}

La investigación realizada acerca del concepto de esquema corporal de niñas y niños con y sin necesidades educativas especiales (nee) de preescolar, segundo y cuarto grados, proporciona información relevante sobre la construcción de este concepto.

La mayor parte de las y los estudiantes con necesidades educativas especiales, de los tres niveles educativos, expresa conceptos de cuerpo relacionados con las ca- racterísticas y las cualidades corporales, aunque algunos también se refieren a habilidades corporales. Es importante resaltar que estos conceptos no presentan diferencias sustanciales con los expresados por las y los estudiantes sin necesidades educativas especiales.

El número de partes del cuerpo mencionadas aumenta a medida que lo hace el nivel educativo y se ve reflejado en las representaciones gráficas de sus cuerpos. No obstante, la mayoría de las y los estudiantes con necesidades educativas

\footnotetext{
${ }^{1}$ Catedrática de la Universidad Nacional. Máster en Psicología de la Universidad de Costa Rica. Excoordinadora e investigadora del Programa de Investigación en Epistemología Genética y Educación del IMEC, Universidad de Costa Rica, exdirectora de la División de Educación Básica del CIDE, Universidad Nacional. Tiene publicaciones en los campos de la Psicología y la Pedagogía.

${ }^{2}$ Máster en Pedagogía con énfasis en Diversidad de los Procesos Educativos. Licenciada en Educación Especial con énfasis en Retardo Mental. Experiencia en Educación Especial y Primaria. Actualmente se desempeña como docente e investigadora en el Centro de Investigación y Docencia en Educación de la Universidad Nacional, tiene publicaciones en los campos de la Integración Escolar de Estudiantes con Necesidades Educativas Especiales y Pedagogía.

${ }^{3}$ Licenciada en Ciencias de la Educación con énfasis en I y II Ciclos. Experiencia docente en educación primaria. Actualmente se desempeña como docente e investigadora en el Centro de Investigación y Docencia en Educación de la Universidad Nacional, tiene publicaciones en Pedagogía.
} 
especiales representaron mayor dificultad en la ubicación de partes del cuerpo con su respectiva lateralidad.

Conforme aumenta el nivel educativo, se identifica que las y los estudiantes con necesidades educativas especiales asumen las labores domésticas y expresan tener mayor dificultad para las actividades escolares. Asimismo, indican que sienten malestar cuando las personas importantes de su entorno les dicen cosas relacionadas con su cuerpo. No obstante, la mayoría de las y los estudiantes con y sin necesidades educativas especiales expresan percepciones positivas relacionadas con su cuerpo y tienen un sentimiento de respeto por sí mismas(os).

Por lo anterior, se recomienda que en los procesos educativos se tomen en consideración los conocimientos previos de las y los estudiantes y se enfatice en la enseñanza de aspectos no sólo referidos a conocimientos del cuerpo, sino relacionados con la imagen corporal. Es valiosa la aplicación de actividades didácticas que permitan la vivencia y construcción de los conceptos estudiados, para prevenir los desajustes que se pueden generar en la adquisición del esquema corporal, o bien, compensar las necesidades educativas derivadas o no de una discapacidad.

\section{Abstract}

This research on the concept of body parts held by preschool, second and fourth grade children with and without special needs provides relevant information on how they construct their body parts concept.

Most of the studied students with special needs show body concepts related to body characteristics and qualities, although some of them also make reference to body skills. It is important to highlight that these concepts are not all too different from those expressed by the group of students without special needs.

The number of body parts listed by students increases with their educational level, as shown in the graphic representations the students made of their bodies. However, most special needs students have greater difficulty in locating body parts and their laterality.

We have found that as students with special needs increase their level of education, they are more likely to take on domestic chores and to find school activities more difficult. They also report feeling uncomfortable when people of higher rank make comments related to their bodies. Even so, most students with and without special needs show a positive perception of their bodies and feel respect for themselves.

Based on these findings, we recommend that all educational processes take into account students' previous knowledge and emphasize not only the teaching of body-related concepts but also concepts related to body image. Teaching activities must also allow students to experience and construct the studied concepts, thus avoiding any wrong learning in the acquisition of concepts about their bodies, or compensating for the educational needs that may derive from a disability.

\section{Palabras claves}

Concepto, esquema corporal, necesidades educativas especiales, preescolar. 


\section{Introducción}

Como parte del trabajo académico de la División de Educación Básica del Centro de Investigación y Docencia en Educación, de la Universidad Nacional, se han venido desarrollando proyectos de investigación, extensión, docencia y producción, en las distintas áreas de especialidad: Educación Preescolar, I y II Ciclos y Educación Especial. Se han realizado proyectos de investigación acerca de conceptos, donde se enmarca el presente artículo y que se proponen no sólo conocer la forma cómo las niñas y los niños construyen los conceptos, sino también fortalecer los procesos de formación de docentes, para brindar un aporte al conocimiento a partir de la realidad costarricense. Los siguientes son algunos de los resultados emanados de una investigación sobre el concepto de esquema corporal realizada por investigadoras de la Unidad Académica.

\section{Consideraciones teóricas}

Como antecedente, cabe señalar que la División de Educación Básica, en concordancia con su preocupación por conocer más de cerca la realidad educativa, desarrolló un estudio relacionado con la construcción de conceptos donde se abordó también el de esquema corporal (León, Pereira y Castro, 2003). No obstante, por la variedad de conceptos que este estudio incluía, fue imposible ahondar en cada uno de ellos, razón por la cual se consideró relevante iniciar un nuevo proyecto que profundizara en los aspectos que ya había sido posible identificar en torno al tema. Se pretende generar mayor conocimiento en esa área específica y fortalecer el trabajo docente con información propia de la población costarricense, para brindar un aporte a la formación de docentes de Preescolar, Educación Especial y I y II Ciclos. La investigación se desarrolló en el 2003 y 2004 con el título de El concepto deesquema corporal en niños de 0 a 10 años con y sin necesidades educativas especiales (Fontana, Pereira y Rojas, 2005).

Los estudios acerca de la construcción de nociones y conceptos en relación con la edad o niveles educativos de las y los estudiantes son escasos en Costa Rica; por esta razón, se plantea la investigación sobre esquema corporal como una forma de acercarse a la comprensión de dicha temática.

La educación, al ser un proceso permanente, dinámico y flexible que se inicia desde la concepción de cada individuo, se da en la familia de manera informal y en la escuela, con un sistema formal, tiene como función el desarrollo integral de todos los miembros de la sociedad. 
En toda acción educativa, se presenta un proceso de interacción entre individuos (docente/discente, madre/hijo, compañero/compañera), cuyo objetivo primordial es propiciar el aprendizaje significativo. Desde este enfoque constructivista, los procesos pedagógicos intencionados cobran importancia, porque buscan promover una evolución y avance en las dimensiones cognoscitivas, socioafectivas y psicomotoras en las y los estudiantes.

Desde esta perspectiva, se asume que las niñas y los niños desde temprana edad, van construyendo diversos conceptos y conocimientos que repercutirán de forma significativa en sus vidas. La adquisición del esquema corporal es uno de ellos, pues es una necesidad básica e indispensable para la construcción de la personalidad. La evolución del esquema corporal está ligada al desarrollo psicomotor global, el cual comprende y diferencia progresivamente las diferentes funciones y procesos de los niveles motores, tónicos, perceptivos, sensoriales y expresivos que están determinados por el aspecto afectivo (Jiménez, 2001).

Por esto, el período escolar viene a ser fundamental para la formación del concepto de esquema corporal, porque es la etapa donde se toma conciencia efectiva del cuerpo mediante las impresiones sensoriales del movimiento y del espacio que rodean al sujeto y que se inician en los primeros seis años de vida. Esta elaboración del esquema corporal tiene elementos constitutivos que se apoyan y se condicionan entre sí, que son las percepciones y control del propio cuerpo, un equilibrio postural, una lateralidad bien definida, una interdependencia entre las partes del cuerpo y su relación con las otras y los otros, siendo el equilibrio, el control y la respiración expresiones e inhibiciones del esquema corporal.

El estudio del esquema corporal y su relación con los procesos educativos y de desarrollo viene realizándose desde hace muchos años, así se encuentran planteamientos como el de Shilder (citado por Riviere, 1966), quien señala que el esquema "es una estructura que funciona a nivel inconsciente" (p. 1). Por lo tanto, es variante, ya sea por discapacidades reales en el cuerpo, por lesiones o desarrollos irregulares de la personalidad, que son el resultado de la interacción recíproca entre el cuerpo y el mundo. Además, indica que la 'envoltura del cuerpo' tiene gran importancia, ya que condiciona a cada sujeto según su sexo, edad, cultura, religión, entre otros aspectos. Es relevante que cada persona sepa que no existe un único ideal de belleza, por lo que a cada ser humano le corresponde aceptar su realidad y tomar decisiones con respecto a ella.

Es importante mencionar que los deseos, los impulsos, las emociones integran el esquema corporal, los cuales aportan no sólo en la construcción del concepto de sí mismo, sino también al de la autoestima. Lo anterior pone en evidencia que el ser humano es una unidad integrada y las dimensiones física, emocional, social y espiritual se fusionan y generan una relación entre los 
sentimientos, actitudes, opiniones, pensamientos y la satisfacción o insatisfacción del cuerpo de cada uno. El mal funcionamiento de uno de los aspectos antes mencionados altera, como consecuencia, las funciones psicosomáticas, mentales, funcionales, neuronales, entre otras. En este sentido, Jiménez (citada por León et al., 2003) indica que si en el desarrollo motriz se presenta alguna perturbación en el esquema corporal y no se controla una parte del cuerpo, se pueden manifestar situaciones variadas, como defectos en la coordinación, lentitud, torpeza, letras deformes, temblores y cuadernos con borrones, entre otras. Como consecuencia, la niña o el niño puede verse amonestada(o) y criticada(o) por parte de docentes, madres o padres de familia, situación que le causa ansiedad y violencia.

El conocimiento del cuerpo es una habilidad que se aprende y le permite al individuo reconocer y controlar el cuerpo y sus partes. La percepción de sí misma(o) y del entorno, producida mediante los sentidos y canales sensoriales, es básica para la adaptación y se irá desarrollando y evolucionando a lo largo de la vida (Ciberarroba, s.f.). Por eso, la estructuración del concepto corporal debe ser sistemáticamente educada desde la infancia, respetando el nivel de maduración de la niña y el niño, para proponer actividades secuenciadas en consonancia con sus capacidades y habilidades que les permitan avances significativos.

Se suele entender por imagen corporal la experiencia subjetiva del cuerpo y los sentimientos respecto a él. En la formación de la imagen corporal participa la percepción que se tiene de sí misma(o), la cual se ha ido configurando a partir de las experiencias con otras personas, sus metas y pertenencia o exclusión de grupos sociales (Lobo, Reyes y Martínez, citados por Arce, 2003). Asimismo, la imagen corporal se ve determinada por las valoraciones procedentes de las personas significativas de su entorno.

$\mathrm{Al}$ respecto, Gurney (citado por Shea y Bauer, 2000) indica que el ser humano es social y busca la aprobación respetando las normas y estándares socialmente establecidos. Por ello, los mensajes verbales y no verbales de las personas que rodean a la niña y al niño (madres y padres, amigas(os), compañeras(os), maestras(os)) y la forma de interpretarlos determinan cómo se percibe a sí misma(o), el valor de sí misma(o) y el grado de competencia.

Lo anterior se relaciona con el hecho de que la primera impresión que se tiene de las(os) otras(os) es mediante su apariencia física y dependiendo de la reacción o actitud que se demuestre, la persona podrá desarrollar un sentimiento de aceptación, negación, confirmación y hasta exageración del concepto que esta tiene de sí misma.

Es importante mencionar que algunas investigaciones relacionadas con la aceptación de la imagen corporal (Intercole, s.f., Mussen y Jones, citados por 
Gratty, 1982) señalan que las personas que tienen dificultades con su cuerpo no se aceptan como una unidad total, generan sentimientos de rechazo por su posición desventajosa en las actividades físicas, algunas se vuelven dependientes y manifiestan actitudes de rebeldía y en la edad adulta conservan restos psicológicos de las diferencias aunque estas hayan desaparecido. Un aspecto importante de considerar es que "(...) el niño que se retarda en el desarrollo físico está expuesto a un ambiente social adverso sobre el desarrollo de su personalidad" (Museen y Jones, citados por Gratty, 1982, p. 176).

El propiciar el acceso a las instituciones de educación regular, de cada estudiante, especialmente de quienes presentan necesidades educativas especiales, es un reto para cualquier sistema educativo. Shea y Bauer (2000) plantean que en la integración de estudiantes con discapacidad, se hace necesario generar procesos educativos y modalidades de apoyo que garanticen su participación en igualdad de oportunidades y que, a la vez, desarrollen las habilidades e imagen social que les permita insertarse en la sociedad como individuos activos. No es haciendo evidente la diferencia como se logra la integración, sino mediante la aceptación del ser humano en todas sus dimensiones y la inserción en igualdad de oportunidades dentro de las capacidades que cada quien tiene.

La escuela tiene una gran influencia en el crecimiento personal y avance académico de todas(os) sus estudiantes, en especial, de las y los que presentan necesidades educativas especiales, ya que sus trabajos constantemente se encuentran expuestos a las valoraciones, tanto de sus compañeras(os) como de sus profesoras(es) y su autopercepción; es decir, de cómo se percibe a sí misma(o), lo cual se nutre en buena medida con lo del entorno escolar. Por lo anterior, su experiencia en ambientes educativos inclusivos positivos contribuye significativamente a fortalecer o disminuir su autoestima y a desarrollar un adecuado o inadecuado autoconcepto.

Shea y Bauer (2000) plantean que la sociedad suele imponer una percepción equivocada a las necesidades educativas especiales del individuo y luego trata de ayudarlo a encontrar formas para superar sus diferencias. Este proceso de identificación le genera una imagen distorsionada de sí misma(o). Cuando una niña o un niño con alguna discapacidad se encuentra incluida(o) en un ambiente en el que sus iguales no coinciden en aspecto con su propia imagen, se ve enfrentada(o) a realizar un trabajo de aceptación y de autovaloración que debe tenerse presente, máxime si se concibe que el autoconcepto y la autoimagen suelen estar referidos a las(os) otras(os) como espejo de la propia existencia y a las características y habilidades que en ellas(os) se ven y que, a la vez, se refieren a la propia persona.

Es importante mencionar los estudios de Bowlby, 1995; Ainsworh, 1967; Fierro, 1990 y Vargas y Polaine-Lorente, 1996 (citados por Meléndez, 2002), 
los cuales demuestran que los patrones de crianza que tienen una posición directiva, específicamente con la figura de vínculo materno, ocasionan una inhibición de la conducta exploratoria y obstaculizan los procesos de desarrollo general de la niña o el niño. Asimismo, esta directividad, o la falta de reciprocidad afectiva de la madre, se asocia con la negación de la autodeterminación de la persona con discapacidad y contribuye a la formación de una representación de fracasada que la acompañará en toda actividad futura que se disponga a realizar. Meléndez (2002) opina que:

Los frecuentes sentimientos de frustración, la hiperactividad, la labilidad emocional, la vulnerabilidad al estrés, las reacciones de ansiedad y el pobre autoconcepto deben clasificarse como secuelas de la indefensión aprendida y de la reacción del ambiente social con respecto a esa indefensión, en lugar de ser atribuidas inequivocadamente al perfil de la persona con discapacidad intelectual (p. 112).

Según Gurney (citado por Shea y Bauer, 2000) cuando las y los estudiantes ingresan a la institución educativa con autoconcepto negativo, las madres y los padres pueden enfrentar sentimientos de incompetencia personal, de desilusión y, dependiendo de la calidad de relación con la hija o el hijo, podrían recoger las señales y mensajes en forma inadecuada y alterar la formación del autoconcepto.

Las niñas y los niños con necesidades educativas especiales suelen tener pocas oportunidades de compartir con otras(os) parecidas(os) a ellas(os). Por eso, cuando llegan a ambientes educativos inclusivos comprenden e identifican sus diferencias y luchan por una aceptación social con el desempeño académico. Esta experiencia podría influir negativamente en su autoestima y provocar incluso sentimientos de incompetencia que afectan su imagen corporal. Esta interesante temática habría de ser abordada mediante procesos de investigación, para lograr una mayor comprensión de este hecho y brindar mejores opciones a las personas con necesidades educativas especiales, que se encuentran insertas en sistemas educativos regulares.

Generalmente, las personas con necesidades educativas especiales cuentan con información restringida acerca de aspectos culturales de su grupo étnico y, por el contrario, saben más de características de los grupos mayoritarios. El currículo escolar, como responsable de una formación integral que beneficie a todas las personas independientemente de sus capacidades, no siempre logra integrar contenidos con temas relacionados con los grupos que representan a personas con necesidades educativas especiales, por ejemplo, algunos de dichos 
contenidos bien podrían ser la historia y logros de personas con pérdidas auditivas o visuales, las biografías de individuos que presentan una discapacidad, entre otros aspectos.

Los autores agregan que los enfoques multiculturales han demostrado que no es adecuado desconocer las diferencias entre individuos, porque estas determinan el desarrollo de la identidad individual y su participación como miembro activo de este grupo en la sociedad.

Se dan evidencias de que si en la infancia las(os) adultas(os) con necesidades educativas especiales tuvieron un acercamiento y se relacionaron con individuos que poseen necesidades educativas especiales similares a las de ellas(os), han podido desarrollar un mejor autoconcepto (Ferguson y Asch, citados por Shea y Bauer, 2000). Si esto es realmente cierto, cabe preguntarse acerca de la efectividad de procesos de integración educativa en los cuales es más posible el señalamiento de la diferencia, que el acercamiento hacia ella. Estos aspectos, a criterio de las autoras del presente artículo, requieren de mayor conocimiento y, por lo tanto, de profundización.

Por otra parte, los nuevos enfoques educativos muestran preocupación por la falta de oportunidades que tienen las y los estudiantes con necesidades educativas especiales, para establecer amistades y relacionarse con otras(os) niñas(os) y adultas(os) con necesidades educativas especiales en ambientes inclusivos, ya que de esta forma se facilitaría el desarrollo de su autoconcepto como grupo que comparte las mismas capacidades, luchas y experiencias.

Meléndez (2002) recomienda fortalecer los programas de atención temprana, con el fin de que orienten su apoyo hacia un enriquecimiento del vínculo entre la madre y su hija(o) y hacia un cambio de actitud potenciadora del desarrollo y preventiva de la indefensión aprendida.

Considerando lo anterior, el centro educativo debe mantener un ambiente abierto y flexible, donde se desarrolle todo tipo de actividades para la estimulación, no sólo de las capacidades intelectuales, sino físicas, afectivas y sociales. Estas actividades contribuirían a compensar necesidades y a nivelar 'desajustes' que se originan en el entorno social y cultural. La adquisición del esquema corporal se debe trabajar integralmente en todas las áreas y contenidos curriculares de cada etapa. Se sugiere iniciar con acciones corporales, pasando luego a interactuar con diversos materiales del entorno, para finalizar con actividades de representación gráfica y simbólica.

Del mismo modo, el ambiente de aula debe ser rico en experiencias, donde la dinámica de las actividades no sólo se circunscriba en el ámbito cognoscitivo, sino que permita el desarrollo físico, social y afectivo. El fortalecimiento del esquema corporal, como parte importante en el desarrollo de todo ser 
humano, debe ser abordado de manera intencional en los procesos educativos, independientemente si las niñas y los niños poseen o no necesidades educativas especiales. Es entonces un asunto de potenciar el desarrollo integral que es imposible de obviar, dadas las implicaciones en el desenvolvimiento adecuado de niñas y niños.

\section{Procedimientos metodológicos}

La investigación realizada fue de tipo exploratorio-descriptivo y se abocó al estudio del concepto de esquema corporal en niñas y niños de preescolar, segundo y cuarto grados con y sin necesidades educativas especiales, en una escuela pública de la provincia de Heredia, adscrita al Programa PROMECUM. El abordaje de ambas poblaciones fue de interés, dada la necesidad de realizar investigaciones integradas entre las disciplinas que conforman la Unidad Académica, para conocer más de cerca la realidad educativa costarricense y fortalecer la docencia en la formación de educadoras(es) por parte de la División de Educación Básica.

Inicialmente, las niñas y los niños fueron identificadas(os) según la presencia o no de necesidades educativas especiales. Cabe destacar que entre las necesidades educativas especiales de estas(os) participantes se encontraban: problemas de aprendizaje y de lenguaje, trastornos emocionales y de comportamiento y tres casos de discapacidad cognitiva con adecuación curricular significativa. Las y los estudiantes se seleccionaron al azar y en el caso de las niñas y los niños que presentaban necesidades educativas especiales todas(os) fueron escogidas(os), para contar con igual número de participantes en ambos grupos. De los tres niveles educativos, participaron 90 estudiantes, 45 sin necesidades educativas ( 24 hombres y 21 mujeres) y 45 con necesidades educativas (24 hombres y 21 mujeres).

La matriz 1 muestra la distribución de las y los participantes en el estudio, según el nivel y el sexo. Asimismo, se especifica quienes presentaban o no alguna necesidad educativa especial.

Para la recolección de datos, se elaboró un instrumento bajo la modalidad de entrevista, para cada uno de los niveles: preescolar, segundo y cuarto grados. Dado que el concepto de esquema corporal está constituido por el esquema mismo (la imagen y el concepto de cuerpo), el instrumento fue construido considerando esos tres criterios. De igual manera, se recopilaron las representaciones gráficas de niñas y niños.

Los instrumentos fueron validados mediante la aplicación piloto en poblaciones similares a las que iban a ser aplicados. También se validaron mediante 
Desglose de participantes, según nivel educativo y sexo

Nivel educativo Preescolar Segundo grado Cuartogrado Total

Con nee $(*)$

Hombres

Mujeres

7

8

7

8

10

Sin nee

Hombres

Mujeres

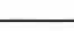

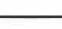

rang

\section{5}

24

21

$(*)$ nee $=$ necesidades educativas especiales.

criterio de jueces - fueron profesoras(es) universitarias(os) con grado de licenciatura o más-, quienes laboraban en la División de Educación Básica en las carreras de I y II Ciclos, Preescolar y Educación Especial.

Una vez validados los instrumentos, se aplicaron de manera individual a cada una(o) de las y los participantes del estudio bajo la modalidad de entrevista clínica, que implicaba que cada investigadora se encontrara con una niña o un niño a la vez, aplicara la entrevista y recopilara la representación gráfica acerca del propio cuerpo.

Como parte de la investigación, se respetan los aspectos éticos, tales como solicitudes de permiso a las instituciones y a las y los participantes, de igual manera se guardó la confidencialidad de las y los informantes.

Se hizo la devolución de los datos a las y los participantes y se procedió a preparar la divulgación de los resultados por tres medios (congresos nacionales, artículos para revista y una guía metodológica dirigida a docentes), en los cuales se destacaron los aspectos básicos encontrados en la investigación, así como sugerencias para trabajar en las aulas el esquema corporal de manera integral.

Para analizar la información, fue transcrita textualmente según el nivel educativo y la presencia o no de necesidades educativas especiales. Se agruparon las respuestas según similitud para aquellos datos que así lo permitían; luego, se formularon categorías de respuesta o cuadros de frecuencia y porcentaje. Aunque la investigación realizada contiene variada información, para efectos del presente artículo se destacarán exclusivamente datos relacionados con las y 
los estudiantes que participaron en la investigación y que presentaban necesidades educativas especiales.

\section{Resultados}

En el gráfico 1 se aprecia el promedio de las partes del cuerpo mencionadas por las y los estudiantes participantes en el estudio. Se deduce que el promedio de partes indicadas por ellas(os) — sea con o sin necesidades educativas especiales - sigue un orden ascendente de preescolar a cuarto grado. Las y los estudiantes con necesidades educativas especiales son las y los que más partes del cuerpo mencionaron en los tres niveles educativos.

Cabe señalar que uno de los aspectos que las y los docentes que atienden estudiantes con necesidades educativas especiales suelen reforzar es justamente el relacionado con el reconocimiento de las diferentes partes del cuerpo, lo cual bien podría estar reflejado en los datos aquí indicados.

\section{Gráfico 1}

Promedio de las partes del cuerpo mencionadas por las niñas y los niños preescolares y escolares con y sin necesidades educativas especiales, según el nivel educativo

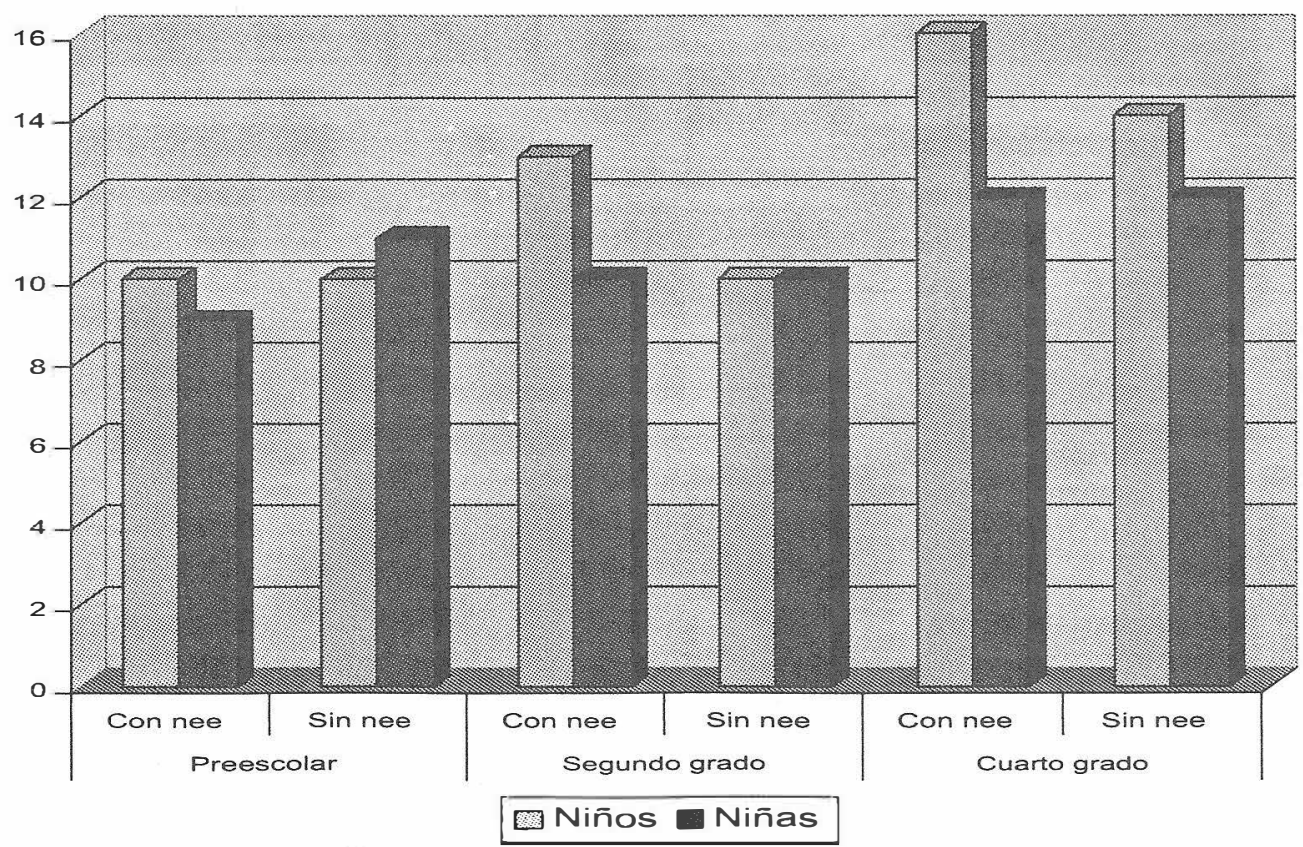




\section{Cuadro 1}

Opiniones de las niñas y los niños preescolares y escolares con y sin necesidades educativas especiales, acerca de lo que es el cuerpo para ellas(os), según frecuencia absoluta y relativa

\begin{tabular}{|c|c|c|c|c|c|c|c|c|c|c|c|c|}
\hline \multirow{3}{*}{$\begin{array}{l}\text { Nivel educativo } \\
\text { Categorías de respuesta }\end{array}$} & \multicolumn{4}{|c|}{ Preescolar } & \multicolumn{4}{|c|}{ Segundo grado } & \multicolumn{4}{|c|}{ Cuarto grado } \\
\hline & \multicolumn{2}{|c|}{ Con nee } & \multicolumn{2}{|c|}{ Sin nee } & \multicolumn{2}{|c|}{ Con nee } & \multicolumn{2}{|c|}{ Sin nee } & \multicolumn{2}{|c|}{ Con nee } & \multicolumn{2}{|c|}{ Sin nee } \\
\hline & $\mathrm{F}$ & $\%$ & $\mathrm{~F}$ & $\%$ & $\mathrm{~F}$ & $\%$ & $\mathrm{~F}$ & $\%$ & $\mathrm{~F}$ & $\%$ & $\mathrm{~F}$ & $\%$ \\
\hline $\begin{array}{l}\text { Características o } \\
\text { cualidades corporales } \\
\text { ("Es algo bueno"). }\end{array}$ & 7 & 41.2 & 10 & 62.5 & 4 & 26.9 & 9 & 56.3 & 4 & 25 & 4 & 22.2 \\
\hline $\begin{array}{l}\text { Habilidades y } \\
\text { destrezas corporales } \\
\text { ("Nos sirve para } \\
\text { movernos"). }\end{array}$ & 4 & 23.5 & - & - & 5 & 33.3 & 5 & 31.3 & 3 & 18.7 & 6 & 33.3 \\
\hline $\begin{array}{l}\text { Elementos que } \\
\text { contienen el cuerpo o } \\
\text { lo conforman } \\
\text { ("Es una capa". } \\
\text { "Es carne o hueso"). }\end{array}$ & 1 & 5.8 & 6 & 37.5 & 5 & 33.3 & - & - & 6 & 37.4 & 3 & 16.7 \\
\hline $\begin{array}{l}\text { Sentimientos o } \\
\text { emociones } \\
\text { ("Es amor". "Algo } \\
\text { que nos hace feliz"). }\end{array}$ & 2 & 11.8 & - & - & - & - & 1 & 6.2 & 1 & 6.3 & 3 & 1637 \\
\hline Creencias religiosas & 2 & 11.8 & - & - & - & - & 1 & 6.2 & 1 & 6.3 & 2 & 11.1 \\
\hline No sabe & 1 & 5.9 & - & - & 1 & 6.8 & - & - & 1 & 6.3 & - & - \\
\hline
\end{tabular}

El cuadro 1 muestra que para las y los participantes en los tres niveles educativos, la referencia al concepto de cuerpo está dada en función de sus características o cualidades corporales. 
En orden descendente se dan respuestas referidas a las habilidades o destrezas corporales, a los elementos que contiene o conforman el cuerpo, a sentimientos y emociones y a creencias religiosas. Es importante destacar que entre niveles no se presentan diferencias conceptuales relevantes entre las niñas y los niños con y sin necesidades educativas especiales.

\section{Cuadro 2}

Partes del cuerpo señaladas correctamente por las niñas y los niños preescolares y escolares con y sin necesidades educativas especiales, según nivel educativo y frecuencia absoluta

\begin{tabular}{l|c|c|c|c|c|c}
\hline Nivel educativo & \multicolumn{2}{|c|}{$\begin{array}{c}\text { Preescolar } \\
\text { Correcto }\end{array}$} & \multicolumn{2}{c|}{$\begin{array}{c}\text { Segundo grado } \\
\text { Correcto }\end{array}$} & \multicolumn{2}{c}{$\begin{array}{c}\text { Cuarto grado } \\
\text { Correcto }\end{array}$} \\
\hline Partes señaladas & $\begin{array}{c}\text { Con nee } \\
\text { F }\end{array}$ & $\begin{array}{c}\text { Sin nee } \\
\text { F }\end{array}$ & $\begin{array}{c}\text { Con nee } \\
\text { F }\end{array}$ & $\begin{array}{c}\text { Sin nee } \\
\text { F }\end{array}$ & $\begin{array}{c}\text { Con nee } \\
\text { F }\end{array}$ & $\begin{array}{c}\text { Sin nee } \\
\text { F }\end{array}$ \\
\hline Cuello & 15 & 15 & 15 & 15 & 15 & 15 \\
\hline Codo & 14 & 14 & 15 & 15 & 15 & 14 \\
\hline Muñeca & 15 & 14 & 13 & 14 & 15 & 14 \\
\hline Tobillo & 09 & 11 & 10 & 15 & 15 & 15 \\
\hline Oreja izquierda & 10 & 08 & 11 & 09 & 15 & 12 \\
\hline Hombro derecho & 02 & 13 & 09 & 06 & 15 & 12 \\
\hline
\end{tabular}

En el cuadro 2 se aprecian las partes del cuerpo señaladas correcta o incorrectamente por las y los participantes. Se nota una mayor dificultad cuando estas y estos estudiantes tienen que ubicar el tobillo, la oreja izquierda y el hombro derecho; puede deducirse que al localizar una parte del cuerpo con su respectiva lateralidad, les es más difícil a las y los estudiantes de preescolar o segundo grado, que a las y los de cuarto grado. Si se analizan los datos en general, se nota que a menor nivel educativo se presenta mayor dificultad en la localización de las partes del cuerpo. Cabe destacar que en preescolar y segundo 
grado las niñas y los niños sin necesidades educativas especiales presentan menos partes correctas, lo que hace necesario fortalecer este aspecto.

Para los niveles de preescolar y segundo grado, las niñas y los niños con necesidades educativas especiales tienen mayor dificultad en la localización de las partes del cuerpo como tobillo, oreja y hombro derecho. Lo expuesto refuerza la necesidad de que para niñas y niños, con o sin necesidades educativas especiales, es igualmente necesario fortalecer el conocimiento del cuerpo y sus partes en las dinámicas que se dan en el aula.

\section{Cuadro 3}

Expresiones de las niñas y los niños preescolares y escolares con y sin necesidades educativas especiales acerca de lo que perciben con su cuerpo, según nivel educativo, frecuencia absoluta y relativa

\begin{tabular}{|c|c|c|c|c|c|c|c|c|c|c|c|c|}
\hline \multirow[b]{3}{*}{ Categorías de respuesta } & \multicolumn{4}{|c|}{ Preescolar } & \multicolumn{4}{|c|}{ Segundo grado } & \multicolumn{4}{|c|}{ Cuarto grado } \\
\hline & \multicolumn{2}{|c|}{ Con nee } & \multicolumn{2}{|c|}{ Sin nee } & \multicolumn{2}{|c|}{ Con nee } & \multicolumn{2}{|c|}{ Sin nee } & \multicolumn{2}{|c|}{ Con nee } & \multicolumn{2}{|c|}{ Sin nee } \\
\hline & $\mathrm{F}$ & $\%$ & $\mathrm{~F}$ & $\%$ & $\mathrm{~F}$ & $\%$ & $\mathrm{~F}$ & $\%$ & $\mathrm{~F}$ & $\%$ & $\mathrm{~F}$ & $\%$ \\
\hline $\begin{array}{l}\text { Percepciones táctiles } \\
\text { (tocar cosas, } \\
\text { calor, frío) }\end{array}$ & 20 & 55.6 & 14 & 53.8 & 12 & 40 & 11 & 52.4 & 17 & 43.6 & 13 & 59.1 \\
\hline $\begin{array}{l}\text { Sensaciones desa- } \\
\text { gradables (golpes, } \\
\text { dolor, vómito, } \\
\text { pellizcos) }\end{array}$ & 14 & 38.8 & 9 & 34.6 & 13 & 43.3 & 10 & 47.6 & 12 & 30.8 & 6 & 27.3 \\
\hline $\begin{array}{l}\text { Sentimientos / entida- } \\
\text { des (a Dios, alegría, } \\
\text { amor, tristeza) }\end{array}$ & 1 & 2.8 & - & - & 5 & 16.7 & - & - & 6 & 15.4 & 2 & 9.1 \\
\hline $\begin{array}{l}\text { Sensaciones agradables } \\
\text { (acariciar, cosas } \\
\text { bonitas, abrazos) }\end{array}$ & 1 & 2.8 & 2 & 7.8 & - & - & - & - & 4 & 10.2 & 1 & 4.5 \\
\hline No sabe & - & - & 1 & 3.8 & - & - & - & - & $\therefore$ & - & - & - \\
\hline
\end{tabular}


Al analizar los datos en forma global, la mayoría de las niñas y los niños con y sin necesidades educativas especiales, en los tres niveles educativos, menciona que con su cuerpo sienten diferentes percepciones táctiles. Otro grupo significativo de estudiantes dijo que percibe sensaciones desagradables como dolor, golpes, pellizcos, vómito y temblores.

Un grupo menor de estudiantes indicó que percibe con su cuerpo sentimientos y emociones como amor, alegría, tristeza y sensaciones agradables como caricias, abrazos, cosas bonitas y a entidades como Dios.

De acuerdo con la información aportada por este cuadro, es notorio que las sensaciones desagradables son las que refieren con mayor frecuencia y pareciera, entonces, que forman parte de la vida cotidiana de las y los estudiantes entrevistadas(os), especialmente las y los que presentan necesidades educativas especiales, ya que fue este grupo quien manifestó percibir en mayor frecuencia este tipo de sensaciones.

Este hecho llama la atención, pues refiere a una doble situación de dolor, no sólo vivir con algún tipo de discapacidad, sino también enfrentar en sus vivencias familiares, sociales y educativas, situaciones que les generan desagrado.

\section{Cuadro 4}

Acciones que pueden realizar muy bien con su cuerpo las niñas y los niños de preescolar, segundo y cuarto grados con y sin necesidades educativas especiales, por frecuencia absoluta y relativa

\begin{tabular}{l|c|c|c|c|c|c|c|c|c|c|c|c}
\hline Nivel educativo & \multicolumn{4}{|c|}{ Preescolar } & \multicolumn{3}{c|}{ Segundo grado } & \multicolumn{4}{c}{ Cuarto grado } \\
\hline Categorías de respuesta & Con nee & \multicolumn{2}{|c|}{ Sin nee } & Con nee & \multicolumn{2}{c}{ Sin nee } & \multicolumn{2}{c}{ Con nee } & \multicolumn{2}{c}{ Sin nee } \\
\hline & F & $\%$ & F & $\%$ & F & $\%$ & F & $\%$ & F & $\%$ & F & $\%$ \\
\hline Actividades recreativas & 16 & 66.7 & 17 & 40.5 & 11 & 34.4 & 4 & 14.8 & 13 & 37.2 & 2 & 13.3 \\
\hline Movimientos / acciones & 5 & 20.8 & 15 & 25.7 & 5 & 15.6 & 9 & 33.3 & 8 & 22.9 & 7 & 46.7 \\
\hline $\begin{array}{l}\text { Destrezas de } \\
\text { desplazamiento }\end{array}$ & - & - & - & - & 6 & 18.8 & 9 & 33.3 & 4 & 11.4 & 6 & 10 \\
\hline Actividades escolares & 3 & 12.5 & 6 & 14.3 & 7 & 21.8 & 3 & 11.2 & 6 & 17.1 & - & - \\
\hline Labores domésticas & - & - & 4 & 9.5 & 3 & 9.4 & 2 & 7.4 & 4 & 11.4 & - & - \\
\hline
\end{tabular}


En el cuadro 4 se aprecia que la mayoría de las niñas y los niños de preescolar, segundo y cuarto grados con y sin necesidades educativas especiales opina que lo que pueden realizar muy bien con su cuerpo, son las actividades recreativas como jugar bola, correr o bailar. Otro grupo considerable de estudiantes indica que puede hacer muy bien diferentes acciones y movimientos tales como saltar, pelar o tocar. Por lo tanto, se afirma que las actividades recreativas son una parte fundamental para el desarrollo del esquema corporal, porque permiten la adquisición de mayores destrezas; asimismo, ayudan a compensar necesidades o desajustes que se originan en el entorno social y cultural, tal como lo indica la revisión teórica.

Un grupo menor indica que hace muy bien las actividades escolares y que puede realizar labores domésticas.

Es importante resaltar que las y los estudiantes que presentan necesidades educativas especiales, de segundo y cuarto grados, son quienes, en su mayoría, hacen referencia a que realizan labores domésticas. Mientras que para el nivel preescolar, las y los estudiantes sin necesidades educativas especiales son quienes realizan labores domésticas. De lo anterior se deduce que a mayor nivel educativo, las y los estudiantes con necesidades educativas especiales, asumen más las labores domésticas.

El cuadro 5 muestra que las niñas y los niños entrevistadas(os) presentan mayor dificultad para realizar diferentes acciones y actividades recreativas. En orden descendente, mencionan las labores domésticas y escolares, siendo el grupo de estudiantes que presenta necesidades educativas especiales, el que expresó tener mayor dificultad en este tipo de actividades. Esto tiene relación con el cuadro anterior, porque a mayor nivel educativo, las(os) alumnas(os) con necesidades educativas especiales asumen las labores domésticas y presentan mayor dificultad en las labores escolares.

El cuadro 6 revela que, mayoritariamente, las apreciaciones de las y los estudiantes entrevistadas(os) acerca de cómo es su cuerpo se enfocan en sus cualidades (bonito, bueno, fuerte). Otro grupo significativo indica que son las características físicas (tamaño, grosor y color). De las anteriores respuestas se puede inferir que las y los estudiantes con o sin necesidades educativas especiales, manifiestan percepciones positivas de su cuerpo considerando sus cualidades y tienen un sentimiento de respeto hacia sí mismas(os). Es importante indicar que el grupo de estudiantes con necesidades educativas especiales es el que aportó mayor cantidad de respuestas. 


\section{Cuadro 5}

Habilidades que les cuesta realizar con su cuerpo a las niñas y los niños de preescolar, segundo y cuarto grados con y sin necesidades educativas especiales por frecuencia absoluta y relativa

\begin{tabular}{|c|c|c|c|c|c|c|c|c|c|c|c|c|}
\hline Nivel educativo & & Pree & colc & & & gund & $\operatorname{grc}$ & & & uarto & grac & \\
\hline & & & & nee & & nee & & nee & & nee & & nee \\
\hline Categorías de respuesta & $\mathrm{F}$ & $\%$ & $\mathrm{~F}$ & $\%$ & $\mathrm{~F}$ & $\%$ & $\mathrm{~F}$ & $\%$ & $\mathrm{~F}$ & $\%$ & $\mathrm{~F}$ & $\%$ \\
\hline $\begin{array}{l}\text { Movimientos / } \\
\text { acciones (bailar / } \\
\text { subir árboles) }\end{array}$ & 3 & 12 & 4 & 23.5 & 9 & 42.9 & 3 & 30 & 4 & 19.1 & 10 & 55.6 \\
\hline $\begin{array}{l}\text { Actividades } \\
\text { recreativas (jugar } \\
\text { bola / hacer ejercicios) }\end{array}$ & 4 & 16 & 5 & 29.4 & 5 & 23.8 & 7 & 70 & 1 & 4.8 & 2 & 11.1 \\
\hline $\begin{array}{l}\text { Actividades escolares } \\
\text { (escribir/ estudiar) }\end{array}$ & 4 & 16 & 4 & 23.5 & 3 & 14.3 & - & - & 10 & 47.6 & 2 & 11.1 \\
\hline $\begin{array}{l}\text { Labores domésticas } \\
\text { (lavar / limpiar) }\end{array}$ & 9 & 36 & 2 & 11.8 & 4 & 19 & - & - & 2 & 9.5 & - & - \\
\hline Ninguna habilidad & 3 & 12 & 1 & 5.9 & - & - & - & - & 2 & 9.5 & 4 & 22.2 \\
\hline $\begin{array}{l}\text { Emociones (controlar } \\
\text { carácter) }\end{array}$ & - & - & - & - & - & - & - & - & 2 & 9.5 & - & - \\
\hline $\begin{array}{l}\text { Manejo de aparatos / } \\
\text { instrumentos (usar la } \\
\text { computadora / cortar } \\
\text { madera) }\end{array}$ & 1 & 4 & 1 & 5.9 & - & - & - & - & - & - & - & - \\
\hline $\begin{array}{l}\text { Aseo e higiene } \\
\text { personal }\end{array}$ & 1 & 4 & - & - & - & - & - & - & - & - & - & - \\
\hline
\end{tabular}




\section{Cuadro 6}

Apreciaciones acerca de cómo es el cuerpo, expresadas por las niñas y los

niños de preescolar, segundo y cuarto grados, con y sin necesidades educativas especiales, según frecuencia absoluta y relativa

\begin{tabular}{|c|c|c|c|c|c|c|c|c|c|c|c|c|}
\hline Nivel educativo & & Prees & cola & & & gunc & $g r$ & & & uarto & gra & \\
\hline & Con & nee & & nee & & nee & & nee & Cor & nee & & nee \\
\hline Categorías de respuesta & $\mathrm{F}$ & $\%$ & $\mathrm{~F}$ & $\%$ & $\mathrm{~F}$ & $\%$ & $\mathrm{~F}$ & $\%$ & $\mathrm{~F}$ & $\%$ & $\mathrm{~F}$ & $\%$ \\
\hline $\begin{array}{l}\text { Apreciaciones referi- } \\
\text { das a cualidades del } \\
\text { cuerpo (bonito, feo, } \\
\text { fuerte) }\end{array}$ & 24 & 64.9 & 10 & 66.7 & 11 & 50 & 8 & 44.4 & 17 & 70.8 & 11 & 73.3 \\
\hline $\begin{array}{l}\text { Apreciaciones referi- } \\
\text { das a características } \\
\text { físicas del cuerpo } \\
\text { (grosor, tamaño, color) }\end{array}$ & 12 & 32.4 & 5 & 33.3 & 11 & 50 & 10 & 55.6 & 7 & 29.2 & 4 & 26.7 \\
\hline $\begin{array}{l}\text { Apreciaciones del } \\
\text { cuerpo relacionadas } \\
\text { con creencias religiosas }\end{array}$ & 1 & 2.7 & - & - & - & - & - & - & - & - & - & - \\
\hline
\end{tabular}

En el cuadro 7 se aprecia que para los tres niveles educativos, las niñas y los niños entrevistadas(os) refieren sentimientos de alegría, felicidad y de bienestar cuando otras personas les expresan frases positivas en relación con su cuerpo. En orden descendente, se dan respuestas relacionadas con sentimientos de malestar, tristeza, enojo y vergüenza cuando les dicen frases negativas.

Si se consideran los datos por nivel educativo, las niñas y los niños de cuarto grado con necesidades educativas especiales manifestaron mayor malestar ante las observaciones de otras personas acerca de su cuerpo. Es posible pensar que dichos sentimientos están relacionados con los cambios físicos experimentados en esta etapa, específicamente, con el tamaño de su cuerpo.

Por otra parte, las niñas y los niños de preescolar son quienes más sentimientos de alegría y felicidad expresaron. Los datos anteriores corroboran lo que la teoría indica en cuanto a que las apreciaciones de las otras personas 


\section{Cuadro 7}

Sentimientos y emociones positivas y negativas, que expresan sentir las niñas y los niños preescolares y escolares, con y sin necesidades educativas especiales cuando otras personas dicen cosas acerca de su cuerpo, según frecuencia absoluta y relativa

\begin{tabular}{|c|c|c|c|c|c|c|c|c|c|c|c|c|}
\hline \multirow{3}{*}{$\begin{array}{l}\text { Nivel educativo } \\
\\
\text { Sentimientos y emo- } \\
\text { ciones positivas y } \\
\text { negativas sentidas por } \\
\text { niñas y niños, cuando } \\
\text { otros dicen cosas } \\
\text { acerca de su cuerpo }\end{array}$} & \multicolumn{4}{|c|}{ Preescolar } & \multicolumn{4}{|c|}{ Segundo grado } & \multicolumn{4}{|c|}{ Cuarto grado } \\
\hline & \multicolumn{2}{|c|}{ Con nee } & \multicolumn{2}{|c|}{ Sin nee } & \multicolumn{2}{|c|}{ Con nee } & \multicolumn{2}{|c|}{ Sin nee } & \multicolumn{2}{|c|}{ Con nee } & \multicolumn{2}{|c|}{ Sin nee } \\
\hline & $\mathrm{F}$ & $\%$ & $\mathrm{~F}$ & $\%$ & $\mathrm{~F}$ & $\%$ & $\mathrm{~F}$ & $\%$ & $\mathrm{~F}$ & $\%$ & $\mathrm{~F}$ & $\%$ \\
\hline $\begin{array}{l}\text { Alegría y felicidad } \\
\text { ("Me siento feliz") }\end{array}$ & 6 & 50 & 8 & 47 & 2 & 13.3 & 4 & 26.7 & 1 & 4.8 & - & - \\
\hline $\begin{array}{l}\text { Bienestar ("Se siente } \\
\text { bien cuando dicen } \\
\text { cosas bonitas") }\end{array}$ & 3 & 25 & 1 & 5.9 & 2 & 13.3 & 3 & 20 & 7 & 33.3 & 4 & 25 \\
\hline $\begin{array}{l}\text { Malestar ("Se siente } \\
\text { mal cuando dicen } \\
\text { cosas feas") }\end{array}$ & - & - & 1 & 5.9 & 4 & 26.7 & - & - & 9 & 42.8 & 4 & 25 \\
\hline $\begin{array}{l}\text { Tristeza (“Me siento } \\
\text { triste”) }\end{array}$ & - & - & 1 & 5.9 & 6 & 40 & 1 & 6.7 & 1 & 4.8 & 1 & 6.3 \\
\hline $\begin{array}{l}\text { Enojado o enfadado } \\
\text { ("Me dan ganas de } \\
\text { pegarle") }\end{array}$ & 1 & 8.3 & - & - & - & - & - & - & 1 & 4.8 & 1 & 6.3 \\
\hline $\begin{array}{l}\text { Sentimiento de que } \\
\text { crece ("Siento que } \\
\text { me hago grande") }\end{array}$ & 1 & 8.3 & 2 & 11.8 & - & - & - & - & - & - & - & - \\
\hline Vergüenza & - & & 1 & 5.9 & - & - & - & - & - & - & - & - \\
\hline Nada & - & - & 2 & 11.8 & 1 & 6.7 & 2 & 13.3 & 2 & 9.5 & 1 & 6.3 \\
\hline No responde & 1 & 8.3 & 1 & 5.9 & - & - & 5 & 33.3 & - & - & 5 & 31.1 \\
\hline
\end{tabular}


sobre el cuerpo, producen satisfacción o insatisfacción y afectan, en forma directa, la imagen corporal.

\section{Conclusiones y recomendaciones}

La mayor parte de las y los estudiantes con necesidades educativas especiales, de los tres niveles educativos, expresa conceptos de cuerpo relacionados con las características y las cualidades corporales, aunque algunos también refieren a habilidades corporales. Es importante resaltar que estos conceptos no presentan diferencias sustanciales con los expresados por las y los estudiantes sin necesidades educativas.

El número de partes del cuerpo mencionadas por los grupos estudiados aumenta a medida que lo hace también el nivel educativo y esto se ve reflejado en las representaciones gráficas de sus cuerpos. No obstante, la mayoría de las y los estudiantes con necesidades educativas especiales son las y los que presentaron mayor dificultad en la ubicación de partes del cuerpo con su respectiva lateralidad.

Conforme aumenta el nivel educativo, las y los estudiantes con necesidades educativas especiales asumen las labores domésticas y expresan tener mayor dificultad para las actividades escolares. Asimismo, indican que sienten mayor malestar cuando las personas importantes de su entomo les dicen cosas relacionadas con su cuerpo. Sin embargo, la mayoría de las y los estudiantes con y sin necesidades educativas especiales expresan percepciones positivas relacionadas con su cuerpo y tienen un sentimiento de respeto por sí mismas(os).

Otro aspecto interesante es que las y los estudiantes con necesidades educativas especiales expresaron mayor cantidad de respuestas en la mayoría de las variables que fueron estudiadas, lo cual se destaca en varios de los gráficos y los cuadros.

A partir de las conclusiones expresadas, cabe recomendar que en los procesos educativos, independientemente de si niñas y niños poseen o no necesidades educativas especiales, se deben tomar en consideración sus conocimientos previos y enfatizar en la enseñanza aspectos no sólo referidos a conocimientos del cuerpo, sino relacionados con la imagen corporal.

Por otra parte, sería también valiosa la aplicación de actividades didácticas que permitan la vivencia y construcción de los conceptos estudiados y, de esta forma, prevenir los desajustes que se pueden generar en la adquisición del esquema corporal, o bien, compensar las necesidades educativas derivadas o no de una discapacidad. 
De igual manera, es importante en el futuro indagar las repercusiones de los factores como herencia, ambiente, familia, habilidades, destrezas, autoestima y características de los ambientes socioculturales en que se desarrollan las y los estudiantes con necesidades educativas especiales en la conformación del esquema corporal, ya que en los procesos de desarrollo cobran importancia la familia, la comunidad y la institución educativa.

Debe considerarse el tema de los sentimientos y las emociones de desagrado que están experimentando niñas y niños acerca de su cuerpo y el impacto que los comentarios y las percepciones que las(os) otras(os) tienen en su formación. Esta temática es necesario retomarla en la formación de futuras(os) docentes y en la atención y capacitación permanente de quienes ya se encuentran en servicio. Si se promueve que la escuela debe velar por el desarrollo integral de niñas y niños, este aspecto entonces se debe considerar. 


\section{Referencias}

Arce, L. (2003). El uso del libro de aprestamiento para niños del Ciclo de Transición que asisten a jardines infantiles públicos, según la percepción de los docentes del circuito 04 de la provincia de San José. Tesis de Licenciatura. Universidad Nacional.

Ciberarroba. (s.f.) Contenidos psicomotores. En línea: http//www.ciberarroba.com/psicomot/contenidos.html

Fontana, A., Pereira, Z. y Rojas, D. (2005). El concepto de esquema corporal en niños de 0 a 10 años con o sin necesidades educativas especiales. Informe final de investigación. Heredia: División de Educación Básica, Universidad Nacional.

Gratty, B. (1982). Desarrollo perceptual y motor en los niños. Barcelona: Editorial Paidós.

Intercole. (s.f.). La imagen corporal. En línea: http//www.intercole.net/portalcnsp/ organizacion/weborientacion/orientacion/laimagencorporal.htm

Jiménez, F. (2001). Talleres de actividades para el desarrollo del esquema corporal. Barcelona: Editorial CEAC.

Jiménez, L. (1978). Esquema corporal. Tesis presentada en la Escuela de Educación, Universidad Nacional.

León, A. T., Pereira, Z. y Castro, M. (2003). Análisis de los procesos cognitivos y pedagógicos que se aplican en el aprendizaje de cinco conceptos básicos del programa de Educación Preescolar del Ministerio de Educación Pública y el desarrollo de estos conceptos en $2^{\circ}$ y $4^{\circ}$ nivel de la Educación General Básica. Informe final de investigación. Heredia: División de Educación Básica, CIDEUniversidad Nacional.

Meléndez, L. (2002). La inclusión escolar del alumno con discapacidad intelectual. Bogotá: Creaciones Altemativas GLARP-IIPD.

Riviere, P. (1966). (Sin título). Disponible: En línea: http/www.esporaldidáctica.com.ar/ 12-9-66.htm/

Shea, T. y Bauer, A. (2000). Educación Especial. Un enfoque ecológico. México: McGRAW-HILL. 